\title{
HEPATITE B: CONHECIMENTO E MEDIDAS DE BIOSSEGURANÇA E A SAÚDE DO TRABALHADOR DE ENFERMAGEM
}

\author{
Hepatitis B: knowledge and measures of biosafety \\ and the health of the nursing worker \\ Hepatitis B: conocimiento y medidas de bioseguranza \\ y la salud del trabaj ador de enfermería
}

Joziane Pinheiro ${ }^{1}$

Regina Célia Gollner Zeitoune ${ }^{2}$

\begin{abstract}
Resumo
0 estudo teve como objetivos: descrever o conhecimento dos profissionais de enfermagem acerca da doença hepatite B; analisar as medidas de biossegurança com relação à hepatite B utilizadas pelos profissionais de enfermagem; e discutir as implicações do conhecimento acerca da hepatite B e as medidas de biossegurança para a saúde do trabalhador de enfermagem. 0 estudo teve amostra de 44 funcionários, representando 100\% dos profissionais de enfermagem do setor de clínica médica de um hospital militar do Município do Rio de janeiro. Constatou-se que a maioria dos profissionais de enfermagem desconhecia as formas de transmissão da hepatite $B$; um número significativo de profissionais de enfermagem não havia recebido treinamento de como proceder caso houvesse um acidente com material perfurocortante; o conhecimento das medidas de biossegurança não estava presente em toda equipe, nem todos as usavam de forma rotineira. Concluímos que os resultados indicam que alguns profissionais estariam expostos ao risco de contrair a hepatite B caso ocorresse acidente com material perfurocortante.
\end{abstract}

Palavras-chave: Saúde do Trabalhador. Enfermagem. Hepatite B.

\begin{abstract}
The Study has as purpose to describe the knowledge of nursing professionals about the hepatitis B disease; to analyze the biosafety measures about hepatitis $B$ used by the nursing professionals; and to argue the knowledge implications about hepatitis $B$ and the biosafety measures for the health of the nursing worker. The study has as sample 44 workers, represented $100 \%$ of the nursing professionals in the medical clinic sector in a military hospital of Rio de Janeiro City. The variables used were: knowledge about hepatitis $B$ and the biosafety measures. Results: the majority of the nursing professionals didn't know the ways of hepatitis B transmission; a significant number of nursing professionals didn't had received training about how to proceed in case of accident with perforating material; the knowledge of the biosafety measures weren't present in the whole staff, neither everybody used it in a routine way. Conclusion: the results indicated that some professionals would be exposed to the risk to acquire hepatitis $B$ in case of accident with perforating material.
\end{abstract}

Keywords: Occupational Health. Nursing. Hepatitis B.

\section{Resumen}

El estudio tubo como objetivo describir el conocimiento de los profesionales de enfermería acerca de la enfermedad hepatitis B; analizar las medidas de bioseguranza con relación a la hepatitis $B$ usada por los profesionales de enfermería; y discutir las implicaciones del conocimiento acerca de la hepatitis By las medidas de bioseguranza para la salud del trabajador de enfermería. El estudio tubo una muestra de 44 funcionarios, representando $100 \%$ de los profesionales de enfermería del sector de clínica médica de un hospital militar de la Ciudad del Rio de Janeiro. Las variables usadas fueran: conocimiento sobre hepatitis $B$ y medidas de bioseguranza. Resultados: la mayoría de los profesionales de enfermería desconocía las formas de transmisión de la hepatitis B; un número significativo de profesionales de enfermería no recibió entrenamiento de cómo proceder si hubiera un accidente con material perforocortante; el conocimiento de las medidas de bioseguranza no estuvo presente en todo el equipo, ni todos las usaban de forma rutinera. Conclusión: los resultados indican que algunos profesionales estarían expuestos al risco de contraer hepatitis B caso ocurriese accidente con material perforocortante. 


\section{INTRODUÇÃO}

0 ambiente hospitalar, como outros cenários de trabalho, também oferecem riscos quando da exposição dos profissionais de saúde e demais trabalhadores a uma diversidade de materiais, especialmente os biológicos. Acredita-se que as atividades laborais exercidas, como, por exemplo, no setor de clínica médica, se constituem fonte de riscos ocupacionais. A natureza do trabalho exige momentos de muita atenção na execução das tarefas, o que pode fazer com que o profissional esqueça de si mesmo e de sua segurança.

Isto porque a formação do profissional de saúde ainda é especialmente voltada para que ele adquira conhecimentos que sejam aplicados aos pacientes. Existe uma distância entre o cuidado ao paciente e o autocuidado do profissional que cuida. Esta dicotomia dificulta a promoção da saúde do trabalhador da saúde. 0 conhecimento recebido na condição de aluno e após formação para realização da prevenção e tratamento das doenças não pode estar direcionado somente para o paciente, e sim, também, para o profissional de saúde.

Corroborando Guimarães Júnior ${ }^{1}$, que concorda com Granovski e loshimoto $0^{5}$ ao afirmar que nos EUA 1.200 pessoas que trabalham na área de saúde são infectadas por ano, o centro de controle de doenças estimou que a infecção dos trabalhadores na área de saúde implica 600 internações hospitalares e 250 mortes por ano, reforçando que, no que se refere em particular à infecção, a hepatite B é muito mais comum em profissionais de saúde do que na população em geral.

Quanto aos profissionais da saúde, a equipe de enfermagem é uma das principais categorias sujeitas a exposições a material biológico ${ }^{3}$. Esse número elevado de exposições relaciona-se com o fato de ser o maior grupo nos serviços de saúde e ter mais contato direto na assistência aos clientes, e também com o tipo e a freqüência de procedimentos realizados.

Desta forma, pode-se inferir que a equipe de enfermagem está contribuindo para este alto número de profissionais de saúde que estão adquirindo hepatite B, número maior que da população em geral citada por Guimarães Júnior ${ }^{1}$.

A análise dos riscos ocupacionais demanda de um conhecimento prévio do processo de trabalho a fim de identificar riscos nele existentes e aqueles advindos dos próprios trabalhadores. Somando-se aos riscos inerentes à profissão, outros poderão ser gerados em virtude do desconhecimento do profissional em evitar danos à saúde. Portanto, ao reconhecê-los, passam-se a analisar, de forma mais precisa, as condições de trabalho, de imunidade do trabalhador, entre outras, que irão influenciar o homem no contexto laboral. 0 conhecimento do perfil imunológico dos profissionais permitirá tomar medidas prévias de prevenção a acidente de trabalho, caso o profissional de saúde desconheça os riscos inerentes a sua profissão, tais como as questões relacionadas ao contato com material biológico.

0 profissional de enfermagem durante o cuidado ao outro não deve esquecer de cuidar de si, e isto não deverá acontecer somente após adquirir uma doença. Ele deverá saber que atua em uma profissão considerada de grande risco a acidentes com material perfurocortante. Diferentemente do que ocorre em outras profissões, esses riscos não são imediatos, pois este profissional estará sujeito a um dano que pode ser percebido meses ou anos após a exposição ao risco, como é o caso da hepatite B.

As ações de saúde para com o próprio trabalhador devem estar integradas com a saúde do cliente, uma vez que os riscos gerados podem afetar também o paciente. Entende-se que haverá preocupação por parte da equipe de enfermagem em motivar a utilização dos recursos disponíveis, como o conhecimento acerca das doenças transmissíveis, prevenindo a contaminação com o vírus da hepatite B, e o "empoderamento" desta clientela, característica da proposta de promoção da saúde, tornando-a co-responsável neste processo.

Dessa forma, poderia ser adotada a estratégia do "empoderamento" na equipe de enfermagem; a responsabilidade pela resolução dos problemas e tomadas de decisões que tradicionalmente era reservada somente para os supervisores passaria a ser dividida com a equipe. A promoção da saúde da equipe de enfermagem não seria de responsabilidade dos supervisores, mas de todos os componentes da equipe de enfermagem 4 .

Com o intuito de promover a saúde dos trabalhadores da saúde, foi aprovada a Norma Regulamentadora 32 (NR32), para trabalhadores regidos pela CLT, que tem por finalidade a implementação de medidas de proteção à saúde dos trabalhadores dos serviços de saúde. Essa Norma corrobora com as medidas de biossegurança que os profissionais de saúde devem cumprir para prevenção de doenças do trabalho, como a hepatite $B^{5}$.

0 parágrafo desta NR 32, que trata dos riscos ambientais, diz que o programa de prevenção de contaminação com material biológico deve conter a identificação dos riscos biológicos mais prováveis, em função da localização e característica do serviço de saúde, considerando fontes e vias de transmissão, estudos epidemiológicos ou dados estatísticos 5 .

A reflexão procede; contudo, há de se ter em mente a perspectiva em que se vem discutindo a saúde do trabalhador, o que irá contribuir para a fundamentação das reflexões, reivindicações e encaminhamentos das questões nesta área.

Ainda nesta perspectiva, corroborando Farias e Zeitoune ${ }^{6}$, quando elas enfatizam a necessidade de investimentos na formação de profissionais de saúde para que eles se sintam mais confiantes, há de se pensar na possibilidade de a unidade de saúde investir na criação de um serviço de saúde do trabalhador, mostrando ao profissional de saúde que todos os riscos relacionados à sua saúde estão sendo monitorados por este serviço e que a instituição está trabalhando em função da promoção da saúde, bem como criar situações para que os profissionais passem a conhecer os riscos a que estão expostos.

Neste sentido, o estudo teve como objetivos: descrever 0 conhecimento dos profissionais de enfermagem acerca da doença hepatite $B$; analisar as medidas de biossegurança com relação à hepatite $B$ utilizadas pelos profissionais de enfermagem; discutir as implicações do conhecimento acerca da hepatite $B$ e as medidas de biossegurança para a saúde do trabalhador de enfermagem. 


\section{REFERENCIAL TEÓRICO}

A hepatite $B$ provocada por vírus é hoje uma doença bem conhecida do ponto de vista clínico, laboratorial e epidemiológico. Trata-se da mais freqüente forma de hepatite infecciosa, sendo a nona causa de mortalidade no mundo? ${ }^{2}$.

Ferreira e Silveira ${ }^{7}$ citam que o Ministério da Saúde estima que, no Brasil, pelo menos $15 \%$ da população já esteve em contato com o vírus da hepatite $B$ e que $1 \%$ da população apresenta doença crônica relacionada a este vírus.

Ao contrário do vírus da hepatite $\mathrm{A}$ (HAV), o HBV permanece no sangue durante os últimos estágios de um período de incubação prolongado (4 a 26 semanas) e durante episódios agudos de hepatite aguda e crônica ${ }^{8}$. Também está presente em todos os líquidos corporais fisiológicos e patológicos. 0 sangue e os líquidos corporais são os veículos primários de transmissão, e o vírus pode se propagar por contato com secreções corporais, como o sêmen, saliva, suor, lágrimas, leite materno e efusões patológicas.

Cerca de 70\% dos pacientes com hepatite B têm hepatite anictérica ou subclínica, e a doença pode ser mais grave em pacientes co-infectados com outros vírus hepatotrópicos ou com doenças hepáticas subjacentes ${ }^{9}$.

Diante da gravidade da exposição dos profissionais de enfermagem ao vírus da hepatite $B$, é necessário que se tomem medidas de prevenção ${ }^{11}$. É importante a adoção de normas e procedimentos seguros e adequados à manutenção da saúde dos pacientes e dos profissionais, ou seja, monitoramento das medidas de biossegurança.

\section{METODOLOGIA}

0 estudo foi descritivo, exploratório, com abordagem quantitativa, o qual envolveu uma coleta sistemática de informações numérica. 0 local de coleta de dados foi um hospital militar localizado no Município do Rio de Janeiro. 0 número de sujeitos foi de 44 profissionais da equipe de enfermagem.

A técnica para a coleta de dados foi a aplicação de um formulário. Utilizou-se o Termo de Consentimento Livre e Esclarecido conforme a Resolução 196/96 do Conselho Nacional de saúde, atendendo às normas e diretrizes referentes à elaboração deste trabalho no que se refere ao respeito à instituição e aos sujeitos balizados pelos princípios da bioética (autonomia, não-maleficência, justiça e equidade) ${ }^{12}$. Obtevese também 0 aceite da Instituição para realização da pesquisa, tendo-a como local de coleta de dados, e aprovação do Projeto em Comitê de Ética da EEAN/HESFA/UFRJ.

\section{RESULTADOS}

A apresentação e a discussão dos dados procuraram atender os objetivos do estudo e estão assim estruturadas: Conhecimentos dos profissionais de enfermagem acerca da doença hepatite $B$ e as medidas de biossegurança com relação à hepatite $B$ utilizadas pelos profissionais de enfermagem. As implicações dos resultados do estudo para a saúde do trabalhador estão permeando toda a análise dos resultados.

\section{Conhecimentos dos profissionais de enfermagem acerca da doença hepatite B}

\begin{tabular}{|c|c|c|c|}
\hline $\begin{array}{l}\text { Tabela 1: } \\
\text { Conhecimento acerca da Do }\end{array}$ & pença Hepati & tite $B(n$ & \\
\hline $\begin{array}{c}\text { Conhecimento acerca } \\
\text { da Hepatite B }\end{array}$ & Respostas & $\mathrm{Fi}$ & $\%$ \\
\hline Formas de transmissão & Sim & 9 & 20,5 \\
\hline do HBV & Não & 35 & 79,5 \\
\hline Número de doses da & Sim & 30 & 68,2 \\
\hline vacina anti-hepatite $B$ & Não & 14 & 31,8 \\
\hline Necessidade da avaliação & Sim & 12 & 27,3 \\
\hline $\begin{array}{l}\text { da soroconversão da vacina } \\
\text { anti-hepatite B }\end{array}$ & Não & 32 & 72,7 \\
\hline
\end{tabular}

Na Tabela 1 observa-se que a equipe de enfermagem não conhecia as formas de transmissão da hepatite B, pois $79,5 \%$ desta equipe não conseguiram apontar todas as formas de transmissão da hepatite $B$.

Com este resultado há de se pensar nos riscos que estes profissionais estão expostos; ou seja, não conhecendo as formas de transmissão, eles poderão ter comportamentos de risco para a hepatite B. Por exemplo, não utilizar as medidas de biossegurança que englobam desde o uso de EPI, a vacinação em si, o treinamento, entre outras.

Ainda na Tabela 1, pode-se observar que $14(31,8 \%)$ desconheciam o número de doses da vacina anti-hepatite $\mathrm{B}$, preconizado pelo Ministério da Saúde. Por não saberem o número adequado de doses da vacina, eles poderiam estar pensando que pelo fato de terem tomado uma ou duas doses apenas estão imunizados; mas na realidade não estão, pois 0 número adequado é de três doses para obtenção da soroconversão. Assim, há de se dizer que este grupo poderá estar exposto à doença em virtude do estado vacinal incompleto.

Um grande número de enfermidades potencialmente transmissíveis pode acometer os profissionais de saúde, destacando-se as infecções transmitidas pelo sangue, dentre elas a hepatite B. Alguns fatores como a duração e freqüência do contato com o sangue e derivados, bem como a positividade de pacientes para AgHBs, são determinantes na infecção ocupacional pelo vírus da hepatite $\mathrm{B}^{13}$.

Diante disso, o desconhecimento do trabalhador pode levá-lo a não completar o esquema vacinal e tornar-se susceptível a doenças.

0 conhecimento do trabalhador hospitalar em relação à sua saúde, especificamente na abordagem do acidente do trabalho e de doenças profissionais, pode ser considerado uma forma de atenção primária em saúde ocupacional ${ }^{14}$.

Um estudo realizado por Oliveira e Murofuse ${ }^{14}$ demonstrou que os trabalhadores de saúde conhecem os riscos à sua saúde de uma forma genérica. Percebeu-se que o conhecimento demonstrado é fruto da prática cotidiana, e não oriundo da existência de um serviço de saúde ocupacional na instituição. Esse conhecimento, entretanto, não se transforma numa ação segura de prevenção de acidentes e doenças ocupacionais, apontando para a necessidade de uma atuação que venha a 
modificar essa situação. Isso representa um esforço de compreensão deste processo - como e por que ocorre - e desenvolvimento de alternativas de intervenção que levam à transformação em direção à apropriação pelos trabalhadores da dimensão humana do trabalho.

Concordando com a posição anterior, Fernandes et al. ${ }^{15}$ referem que o profissional de saúde está exposto a doenças infecciosas em sua prática diária. A vacinação adequada destes profissionais pode diminuir o risco de morbidade por certas infecções, haja vista que a imunização ativa é uma das prevenções mais eficazes contra doenças imunopreveníveis. 0 risco de aquisição de doenças depende não só do tipo de acidente e da profilaxia pós-exposição existente, mas também da prevalência local de doenças e da susceptibilidade do acidentado.

0 conhecimento das técnicas para prevenção de transmissão de certas doenças infecciosas, como o emprego de cuidados universais ao lidar com pacientes e materiais biológicos, o uso de equipamentos de proteção individual e medidas para não-dispersão e transmissão aérea de certos agentes infecciosos, é um fator de relevância e deveria anteceder ao início da prática clínica.

Nos EUA, em 1982, o Center for Disease Control and Prevention (CDC) recomendava a prevenção do contato direto da pele ou das membranas mucosas com sangue, secreções, excreções e tecidos de pacientes com suspeita ou diagnóstico de AIDS, com base nas observações iniciais de que a doença era causada por um agente transmissível ${ }^{16}$.

Com efeito, as recomendações enfatizavam as mesmas precauções antes indicadas a pacientes sabidamente infectados pelo vírus da hepatite $B$. Estas recomendações, denominadas "Precauções contra sangue e fluidos corporais", incluíam: - a manipulação cuidadosa de instrumentos perfurocortantes contaminados com material biológico, devendo, para efeito, ser utilizado coletor resistente para descarte desses materiais perfurocortantes ou cortantes e evitados o reencapamento de agulhas e a desconexão da agulha da seringa; - uso de luvas e de capotes (aventais) quando existir a possibilidade de contato de sangue, fluidos corporais, excreções, secreções; - lavagem das mãos após a retirada das luvas, antes das saídas do quarto dos pacientes, também sempre que houver exposição de sangue.

Com bases nestas conclusões, o CDC implementou o conceito de "Precauções Universais", que são conjuntos de medidas nas quais estão englobados alguns conceitos das recomendações prévias para a prevenção da transmissão do HIV no ambiente de trabalho, tais como: o uso rotineiro de barreiras de proteção (luvas, capotes, óculos de proteção ou protetores faciais) e precauções necessárias na manipulação de agulhas ou materiais cortantes para prevenir exposições em procedimentos invasivos ${ }^{16}$.

Em 1996, o CDC atualizou as práticas de controle de infecções hospitalares, incluindo a categoria de isolamento de substância corporal, conceituando a prevenção do contato com todos os fluidos corporais, como secreções, excreções com pele não íntegra e membranas mucosas, provenientes de qualquer paciente, o que se tornou mais abrangente do que 0 conceito das Precauções Universais, que eram associadas somente a fluidos corporais que pudessem transmitir o HIV e outros patógenos de transmissão sangüínea ${ }^{16}$.

Quanto à necessidade de avaliação da soroconversão da vacina anti-hepatite $B$, verificou-se que a maioria da amostra $(72,7 \%)$ não achava necessário realizar o teste sorológico anti-HBs.

Não há razão para se determinar a resposta laboratorial de anticorpos à vacinação em crianças, adolescentes e adultos sadios ${ }^{17}$. No entanto, para grupos de risco, imunocomprometidos e para os profissionais de saúde, está indicada a avaliação da soroconversão através da realização do teste sorológico anti-HBs.

Ainda na perspectiva da importância da avaliação da soroconversão em estudos realizados, a hepatite B é uma das doenças que mais causa contaminação por acidente de trabalho em profissionais de saúde. 0 risco de contaminação pelo vírus da hepatite $B(\mathrm{HBV})$ está relacionado, principalmente, ao grau de exposição ao sangue no ambiente de trabalho e também à presença ou não do antígeno HBsAg no cliente-fonte ${ }^{18}$.

Desta forma, o desconhecimento da soroconversão poderá ser um indicativo de risco à doença no caso de um acidente biológico, ou seja, a freqüência com que é possível entrar em contato com sangue ou outros materiais biológicos, incluindo exposições percutâneas e mucocutâneas.

Os profissionais de saúde das áreas cirúrgica, odontológica, enfermagem e aqueles que trabalham em atendimento de emergência são classificados como profissionais de alto risco à exposição com materiais biológicos ${ }^{18}$, justificando a inquietação com relação ao acompanhamento da avaliação da soroconversão.

Reafirmando o dito anteriormente, a equipe de enfermagem é uma das principais categorias profissionais sujeitas à exposição a materiais biológicos ${ }^{18}$. Esta não está somente relacionada à assistência direta a clientes, mas também ao tipo e à freqüência de procedimentos realizados.

Os risco de transmissão do HVB nos profissionais da área de saúde é cerca de três a cinco vezes maior do que nos demais membros da população ${ }^{19}$.

São de causar inquietação as respostas que apontam o desconhecimento acerca da transmissão da doença, uma vez que todos são profissionais de enfermagem possuem este conteúdo na sua formação. Há de se pensar para investigações futuras o que leva o profissional a não apreender informações tão importantes? É pertinente, aqui, refletir sobre a importância do treinamento e educação do trabalhador, resgatando aspectos de promoção da saúde e prevenção de doenças.

Cabe ressaltar que as medidas profiláticas pós-exposição existentes ainda não são totalmente eficazes. Assim, a prevenção das exposições ao sangue ou a outros materiais biológicos é a principal e mais eficaz medida pra evitar a transmissão do vírus da hepatite $B$.

\section{Medidas de Biossegurança e a Hepatite B}

Os acidentes com material biológico são bastante freqüentes entre os profissionais de enfermagem, já que existe uma manipulação deste tipo de material. Ou seja, a equipe de enfermagem está sob constante risco de contaminação por agentes infecciosos.

Por outro lado, o profissional que detém o conhecimento de como agir diante dos acidentes é capaz de tomar as 
providências necessárias até mesmo diante das situações estressantes, uma vez que este tem conhecimento dos riscos à sua própria saúde, inerentes à profissão, e sabe que, ao mesmo tempo em que se propõe a cuidar da saúde dos outros, ele deve cuidar e se preocupar com a própria saúde.

É diante do exposto que a preocupação com as normas de biossegurança se torna cada vez mais constante no contexto da saúde do trabalhador de enfermagem e da saúde como um todo.

As normas de biossegurança são procedimentos que funcionam como um conjunto, no qual a realização incorreta de algum deles compromete a biossegurança. Desta maneira, o não-uso dos equipamentos de proteção individual, bem como a falta dos cuidados gerais e locais a serem tomados quando da exposição a material biológico, traz um risco à saúde do profissional. Desta forma, é necessário conhecer o perfil da equipe de enfermagem quanto ao conhecimento das medidas de prevenção para assim poder intervir.

\section{Tabela 2:}

Medidas de Biossegurança $(n=44)$

\begin{tabular}{|c|c|c|c|}
\hline Medidas de Biossegurança & Respostas & $\mathrm{Fi}$ & $\%$ \\
\hline $\begin{array}{l}\text { Utilização de EPI durante assistência } \\
\text { a clientes }\end{array}$ & $\begin{array}{l}\text { Sim } \\
\text { Não }\end{array}$ & $\begin{array}{l}30 \\
14\end{array}$ & $\begin{array}{l}68,2 \\
31,8\end{array}$ \\
\hline $\begin{array}{l}\text { Recebimento de treinamento/ } \\
\text { orientação de como proceder em caso } \\
\text { de acidente com perfurocortante/ } \\
\text { material biológico }\end{array}$ & $\begin{array}{l}\text { Sim } \\
\text { Não }\end{array}$ & $\begin{array}{l}29 \\
15\end{array}$ & $\begin{array}{l}65,9 \\
34,1\end{array}$ \\
\hline $\begin{array}{l}\text { Conhecimento de como proceder em } \\
\text { caso de acidente com } \\
\text { perfurocortante }\end{array}$ & $\begin{array}{l}\text { Sim } \\
\text { Não }\end{array}$ & $\begin{array}{l}26 \\
18\end{array}$ & $\begin{array}{l}59,1 \\
40,9\end{array}$ \\
\hline
\end{tabular}

Conforme a Tabela 2, a utilização de EPI pela equipe de enfermagem se faz em $68,2 \%$. Este dado confirma o estudo, realizado por Farias e Zeitoune ${ }^{20}$ em um Centro Municipal de Saúde, que mostrou que por diversas vezes foi detectada a nãoutilização de equipamento de proteção individual pelos funcionários.

Enfermeiros, técnicos e auxiliares de enfermagem expõem a sua segurança e saúde às condições de trabalho envolvendo-se em múltiplos riscos e cargas no trabalho que ameaçam a sua sobrevivência. Dentre as cargas apontadas pelos autores, encontram-se as biológicas, geradoras de grande diversidade de doenças ${ }^{13: 9}$.

Estes dados também vêm ao encontro de resultados da pesquisa de Sarquis e Felli ${ }^{20}$ sobre acidentes com instrumentos perfurocortantes, onde encontraram alta freqüência de acidente de trabalho entre os trabalhadores de enfermagem, apontando a não-aderência ao uso de equipamento de proteção individual e constatando a grande exposição aos riscos biológicos e às graves doenças como, por exemplo, a AIDS e a hepatite B.

A manipulação de materiais contaminados com todos os tipos de secreç̃̃es é inerente à própria atividade dos profissionais de enfermagem. 0 grande problema é que esses profissionais muitas vezes os manipulam de forma incorreta, aumentando o risco dos acidentes.

Com vista a esta questão, a Norma Regulamentadora 32 estabelece diretrizes básicas para implantação de medidas de proteção em relação à segurança e à saúde dos trabalhadores, bem como daqueles que exercem atividades de promoção e assistência à saúde em geral.

Neste sentido, vale ressaltar que, gradativamente, a Legislação vem contemplando um conjunto de dispositivos que ultrapassam a mera preocupação com a prevenção e o tratamento dos acidentes do trabalho e doenças ocupacionais, considerando aspectos da saúde relacionados à saúde do trabalhador, na tentativa de dar subsídios para que os trabalhadores tenham ambiente de trabalho que comprometam menos sua saúde.

Pode-se dizer que as estratégias já propostas pela própria legislação para saúde dos trabalhadores possam servir como fonte de saber para repensar a saúde do trabalhador de enfermagem e propor alternativas de mudanças para os comprometimentos hoje existentes.

Ainda na perspectiva das medidas de biossegurança e riscos biológicos, Sêcco ${ }^{22}$ aponta que os profissionais de enfermagem inseridos na dinâmica da assistência hospitalar estão particularmente expostos aos riscos biológicos resultantes dos processos de trabalho desenvolvidos. Os agravantes que predispõem a essas ocorrências é a proximidade física necessária na prestação da assistência, que possibilitam a exposição a material biológico potencialmente contaminado.

A situação não se apresenta como um agente de agravo à saúde do profissional de enfermagem somente naqueles que lidam com clientela portadora de hepatite, mas no grupo como um todo, considerando inicialmente o risco do acidente e provável contaminação do profissional.

Em uma outra circunstância de risco, mas semelhante a do estudo em tela, é a da pesquisa realizada por Macie ${ }^{23} \mathrm{em}$ estudantes de enfermagem, onde se pode observar que $63 \%$ dos estudantes não utilizavam equipamento de proteção individual quando prestavam assistência a pacientes com diagnóstico de tuberculose.

Nesta linha de raciocínio, pode-se inferir que, sendo os profissionais de enfermagem considerados grupo de risco para hepatite B, o uso de equipamento de proteção individual (EPI) não deveria ser desconsiderado por nenhum membro da equipe durante a assistência.

Na possibilidade de compreender, mas não justificar o comportamento do profissional de enfermagem acerca da utilização do EPI, a rotina do serviço de enfermagem tem se mostrado como um dos fatores relacionados aos altos índices de acidentes, assim como à não-utilização do EPI. Figueiredo et al. ${ }^{24}$ acreditam que a rotina do serviço pesada e desgastante minimize o sentimento de pânico gerado no momento do acidente. Contudo, isso não impede o risco de acidente e de contaminação. É preciso criar situação de treinamento e reflexão acerca desse comportamento e reversão dessa situação.

Ainda na Tabela 2, pode-se verificar que $34,1 \%$ da equipe referiu não ter tido nenhum treinamento sobre como proceder em caso de acidentes com material perfurocortante.

Ao analisar a saúde do trabalhador no contexto da Enfermagem, através dos tempos, é possível verificar que estes trabalhadores estão expostos a várias cargas que comprometem a saúde, gerando índices elevados de acidentes de trabalho e doenças relacionadas ao trabalho ${ }^{25}$. 
Em outro estudo, Sarquis ${ }^{26}$ encontrou resultados que mostraram trabalhadores de enfermagem expostos aos riscos em até $35,9 \%$ do total de trabalhadores e sugere que há necessidade de educação do trabalhador, em uma visão prevencionista, em relação aos riscos ocupacionais, assim como de uma reestruturação dos currículos das escolas que formam estes profissionais de saúde. Tal fato vem da possibilidade de reforçar a importância e necessidade de se discutirem tais questões na formação do enfermeiro.

Considerando a magnitude do contexto, pode-se constatar que 0 alto risco ocupacional dos trabalhadores de enfermagem pode ser decorrente do não-cumprimento das normas de segurança devido a uma falta de orientação que deveria ser dada à equipe antes de iniciar sua atividade profissional e, até mesmo, da não-continuidade aos esclarecimentos necessários à prevenção de acidentes no âmbito do trabalho através da educação permanente.

A partir dessa percepção, faz-se necessário compreender melhor essa problemática e buscar a legislação trabalhista, ressaltando as questões da saúde do profissional de enfermagem. Esta análise permite verificar que a legislação trabalhista avançou a partir da promulgação da Constituição Federal (1988), na qual as leis orgânicas foram se atualizando e resultaram em exigências legais transformadas em portarias, leis e decretos que se modificaram, completando-se e se alterando com o objetivo de adequar a saúde do trabalhador ao ambiente de trabalho.

Assim, Carvalho ${ }^{27}$ refere que a maior parte das doenças e acidentes de trabalho poderia ser evitada por meio de programas preventivos de saúde e de segurança no trabalho, bem como com medidas coletivas e individuais de proteção, através do uso de equipamentos de proteção coletiva (EPC) e equipamentos de proteção individual (EPI), que constitui uma barreira protetora para o trabalhador.

Nesse sentido, os cuidados na manipulação e descarte de materiais perfurocortantes deverão ser tomados em conjunto com o uso dos EPI (luvas, gorros, óculos, capotes) que têm como objetivo a redução dos riscos de exposição do profissional de saúde a sangue e fluidos corpóreos.

Para assegurar que haja um menor risco de aquisição ou transmissão de doenças infecciosas para o profissional de saúde, tanto a educação em relação ao emprego correto de proteção individual como a vacinação adequada devem ser realizadas previamente ao ingresso do profissional de saúde em sua prática diária, o que ainda tem ficado a desejar.

A implementação de campanhas educacionais para o profissional de saúde sobre prevenção de doenças transmissíveis nosocomiais (vacinação, uso de equipamento de proteção individual) seria fundamental para diminuir o risco de aquisição e transmissão de certas doenças infecciosas ${ }^{28: 25}$.

Com vistas a estas considerações, Lucena ${ }^{29}$ ressalta que os profissionais da área de enfermagem são os que mais se acidentaram no trabalho. Em seu estudo encontrou que apenas $61,9 \%$ eram vacinados adequadamente contra hepatite $B$.

Outro aspecto investigado no presente estudo foi o procedimento no caso de acidente de trabalho, $41 \%$ da amostra não souberam responder como deveriam proceder nesse caso. 0 fato permite inferir que há necessidade da educação permanente, resgatando esta questão junto aos trabalhadores de enfermagem.

Numa perspectiva reflexiva em dimensão maior, pode-se dizer que muitas pessoas vivem e trabalham em condições prejudiciais à saúde e estão potencialmente expostas a produtos perigosos ${ }^{30}$. Tais problemas muitas vezes transcendem as fronteiras nacionais. A administração e gestão ambientais deveriam proteger a saúde humana dos efeitos adversos diretos e indiretos de fatores biológicos, químicos e físicos, vindo a somar com os esforços institucionais, através dos serviços de saúde do trabalhador, na perspectiva da manutenção da saúde do homem trabalhador.

\section{CONCLUSÃO}

Os resultados permitiram concluir que a maioria dos profissionais não conhecia todas as formas de transmissão do vírus da hepatite $B$, como também não conhecia o número de doses da vacina anti-hepatite $B$.

No caso da prevenção da hepatite B no ambiente de trabalho, são de suma importância a vacinação e 0 conhecimento da imunidade à hepatite $\mathrm{B}$, uma vez que a totalidade dos profissionais de enfermagem ainda não utilizava a medida de biossegurança mecânica como 0 equipamento de proteção individual. Para outras doenças, é necessário estar sempre reiterando as conseqüências do uso inadequado do EPI, em virtude de não termos vacinas para prevenção de determinadas doenças.

Existe uma necessidade de fazer intervenções quanto à prevenção da hepatite $B$ no ambiente de trabalho, pois um número significativo da equipe de enfermagem ainda tem dúvidas quanto ao número de doses da vacina anti-hepatite $B$ que é recomendado pelo Ministério da Saúde e da necessidade da avaliação da soroconversão da vacina anti-hepatite $B$.

Ainda quanto às medidas de biossegurança, foi verificado que os profissionais de enfermagem necessitavam de um treinamento permanente visando aumentar seus conhecimentos quanto à tomada de decisão correta em caso de exposição às secreções corporais, para minimizar as conseqüências desta exposição.

\section{Referências}

1. Guimarães Júnior J. Biossegurança e controle de infecção cruzada. São Paulo (SP): Santos; 2001.
2. Rapparini C. Riscos biológicos e profissionais de saúde [on-line]. [citado 10 mar 2005] Disponível em: <http://www.riscobiologico.org>.

3. Organização Pan-Americana da Saúde - OPAS. Participação comunitária e empoderamento. Conceito. [citado 13 ago 2006] Disponível em: <www.opas.org.br/coletiva/temas.cfm 
4. Martins N, Felix JP, Mamari LSS. NR32 - Para a segurança do profissional de saúde. J Control Infec 2005; ano 15 (59).

5. Granovski N, loshimoto L.M. Situação atual e perspectivas para o controle da hepatite B no Brasil [on-line]. [ citado 05 dez 2004] Disponível em: <http://www.videbulas.com.br.html>.

6. Farias SNP, Zeitoune RCG. A interferência da globalização na qualidade de vida no trabalho: a percepção dos trabalhadores de enfermagem. Esc Anna Nery Rev Enferm 2004; 8 (3)

7. Ferreira CT, Silveira TR. Hepatites virais: aspectos da epidemiologia e da prevenção. Rev Bras Epidemiol 2004; 7(4): 473-87.

8. Ramzi SC, Vinay T, Tucker C. Patologia estrutural e funcional. $6^{\mathrm{a}} \mathrm{ed}$. Rio de Janeiro (RJ): Guanabara Koogan; 2000.

9. Coelho HSM, Alves JG, organizadores. Gastroenterologia- hepatites. Sociedade de Gastroenterologia do Rio de Janeiro.Rio de Janeiro(RJ): Rubio; 2001.

10. Coelho H, Santana T, Silva JC, Silva EF, Genuíno CF, Sidoni M. Avaliação da resposta vacinal contra hepatite $B$ em profissionais de saúde do Instituto Fernandes Figueira. [on-line] 2003. [citado 08 nov 2004]. Disponível em: <http://www.bissegurancahospitalar.com.br /files/ hepab.doc.html>.

11. Oliveira A, Albuquerque CP. Infecção hospitalar: abordagem, prevenção e controle. São Paulo (SP): MEDSI; 1998.

12. Ministério da Saúde (BR). Conselho Nacional de Saúde. Diretrizes e normas reguladoras de pesquisa envolvendo seres humanos. Resolução n 196 de 10 de outubro de 1996. Inf Epidemiol SUS 1996; 5 (2): 14-41.

13. Lopes CLR, Martins RMB, Moggi PS, Silva SA, Teles SA, Yoshida CST. Perfil epidemiológico da infecção pelo vírus da hepatite $B$ em profissionais de saúde das unidades de hemodiálise de Goiânia. Rev Soc Bras Med Trop [on-line] 2001 nov/dez; 34 (6). Disponível em: < http://www..scielo.br/cgi-bin/wxis.exe/eah>.

14. Oliveira BRG, Murofuse NT. Doença ocupacional: estudo sobre o conhecimento do trabalhador hospitalar dos riscos à saúde dos trabalhadores. Rev Latino- am Enfermagem 2001; 9(1).

15. Fernandes GC, et al. Possível susceptibilidade a doenças imunopreveníveis em médicos residentes no Rio de Janeiro. [on-line]. 2000. [citado 02 jul 2006] Disponível em: <http:// www.riscobiologico.or/imunização/trabalhador/htm

16. Garner MN. The hospital infection control pratices advisory committee. Inf Control Hosp Epidemiol 1996; 17(1).

17. Ferreira $A B H$. Novo Dicionário Aurélio: dicionário eletrônico.[CD-ROM]; 2004
18. Rapparini C. Implementação de um programa de vigilância e instituição de quimioprofilaxia pós-exposição ocupacional ao HIV no município do Rio de Janeiro. [dissertação de mestrado] Rio de Janeiro (RJ): Faculdade de Medicina/ UFRJ; 1999.

19. Souza M. Assistência de enfermagem em infectologia. São Paulo (SP): Atheneu; 2000.

20. Farias SNP, Zeitoune RCG. Riscos no trabalho de enfermagem em um Centro Municipal de Saúde. Rev Enferm UERJ 2005; 13(2): 167-73.

21. Sarquis LMM, Felli VEA. Acidentes de trabalho com instrumentos perfuro cortantes entre os trabalhadores de enfermagem. Rev Esc Enferm USP 2002; 3(3): 222-30.

22. Sêcco IAO. Acidentes de trabalho com material biológico na equipe de enfermagem de hospital escola público de Uberaba. São Paulo(SP): Atheneu; 2003.

23. Maciel EL. Infecção por mycobacterium tuberculosis em estudantes de enfermagem: uma incidência através do testePPD. [dissertação de mestrado] Rio de Janeiro (RJ): Escola de Enfermagem Anna Nery/UFR; 1999.

24. Figueiredo RM, et al. Acidentes com risco biológico: a adesão de profissionais de saúde ao seguimento. Anais do $51^{\circ}$ Congresso Brasileiro de Enfermagem. $10^{\circ}$ Congresso Panamericano de Enfermagem; 1999 out. 2-7; Florianópolis (SC), Brasil. Florianópolis (SC):ABEn; 2000. p.358.

25. Sarquis LMM, Cruz EBS. Peduzzi M. Uma reflexão sobre a saúde do trabalhador de enfermagem e os avanços da legislação trabalhista [on-line] 2004 [citado 02 jul 2006]. Disponível em: $<$ www.opas.org.br>.

26. Sarquis LMM. Acidentes de trabalho com instrumentos pérfurocortantes: ocorrências entre os trabalhadores de enfermagem. [dissertação de mestrado] São Paulo (SP): Universidade de São Paulo; 1999.

27. Carvalho GM. Enfermagem do trabalho. São Paulo (SP): EPU; 2001.

28. Cortês G. Imunizações e profissionais de saúde. 2005. [on-line] [citado 02 jul 2006]. Disponível em: <http://www.riscosbiologicos.org/ patogenos/hepatb.htm>.

29. Lucena NO. Estudo da prevalência dos acidentes ocupacionais, envolvendo sangue e fluidos corpóreos, na transmissão do HIV, VHB e VHC, ocorridos com profissionais da saúde na Fundação de Medicina Tropical do Estado do Amazonas. [tese de doutorado] Manaus (AM): Universidade Estadual do Amazonas; 2004.

30. Mendes IAC. Desenvolvimento e saúde: a declaração de Alma-Ata e movimentos posteriores. Rev Latino-am Enfermagem 2004; 12(3): 447-48. 